\title{
Non-Foster Matching Network for a Small, Blade- type Monopole in the VHF Band
}

Fernando Albarracín-Vargas, Daniel Segovia-Vargas

\author{
Vicente Gonzalez-Posadas
}

\begin{abstract}
In many applications, like in aeronautical control and communication systems, there are important requirements for the antenna engineer in terms of size and weight of the antennas. Active matching networks, with non-Foster elements, are an interesting way to face the design of an Electrically Small Antenna (ESA). Using the new sensitivity parameter Sens, a good location for a matching network (MN) is found in the structure itself of a blade-type small monopole. Then, comparative design between a passive reactive and an active non-Foster matching network is presented.
\end{abstract}

\section{INTRODUCTION}

Electrically small antennas (ESA) are structures very difficult to match over significant bandwidths because of the quasi-purely reactive nature of its impedance. The upper bound of the impedance bandwidth, and therefore, the lower bound of the quality factor $Q$ of an ESA, described in [1], imposes an important constraint in size and radiation efficiency of the antenna. On the other hand, if we are working with passive matching networks, an additional limit is imposed by the BodeFano gain-bandwidth criterion. It states that one can achieve a given gain in a very small bandwidth by matching the antenna with lossless capacitors and inductors, or achieve larger bandwidths by including losses in the matching network.

One way to overcome these constraints is including active non-Foster elements in the matching network [2] realized by transistor-based circuits called Negative Impedance Converters (NIC). A NIC is a 2-port active network in which one port presents a negated version of the impedance loading the other port, so non-Foster elements such as negative capacitors or inductors can be implemented.

In this work an blade-type small monopole of $22 \mathrm{~cm} \mathrm{X} 25$ $\mathrm{cm}$ over a substrate of $1.6 \mathrm{~mm}$ in width and $\varepsilon_{\mathrm{T}}=8$, for aeronautical applications, is present to be matched (i.e. $\left|S_{1 I}\right|<6$ $\mathrm{dB}$ ) over a 50-88 MHz (VHF band) using, as a comparative design, both strategies the conventional passive and the active non-Foster matching networks. The new Sens parameter, presented in [3] is used to find the best location for the active matching network over the monopole structure itself through a sensitivity analysis.

\section{SENSITIVITy ANALysis FOR A Two PoRt ANTENNA}

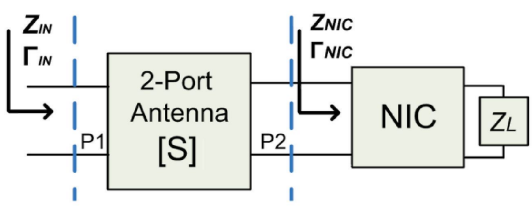

Fig. 1. Two-port antenna approach.

Two-port antenna approach, sketched in Fig. 1, is used to deduce the more convenient port location in our structure [5]. The reflection coefficient at the input, $\Gamma_{\mathrm{IN}}$, is given by (1); as the goal is to have a matched ESA, the input reflection coefficient should be zero over our intended design bandwidth (50-88 MHz). Then, once (1) is set to zero, the optimum reflection coefficient that the NIC must provide, can be extracted as (2):

$$
\begin{gathered}
\Gamma_{I N}=S_{11}+\frac{S_{12} \cdot S_{21} \cdot \Gamma_{N I C}}{1-S_{22} \cdot \Gamma_{N C}}=0 \rightarrow \\
\rightarrow \Gamma_{N I C}^{a n}=\frac{S_{11}}{S_{22} \cdot S_{11}-S_{12} \cdot S_{21}}
\end{gathered}
$$

Therefore, the associated non-Foster impedance, $Z_{N I C}^{a n}$ needed to match the antenna at port 1 can be expressed in terms of the $S$-parameters of the antenna:

$$
Z_{N I C}^{a n}=Z_{0} \cdot\left(\frac{S_{22} \cdot S_{11}-S_{12} \cdot S_{21}+S_{11}}{S_{22} \cdot S_{11}-S_{12} \cdot S_{21}-S_{11}}\right)
$$

When this impedance $z_{\text {NIC }}^{a n}$ is placed at port 2, the reflection coefficient at port $1, \Gamma_{I N}$, is ideally equal to 0 at all the frequencies. In that sense, the sensitivity parameter Sens can be derived from (4) and (5) and understood as how the changes in the NIC impedance, $Z_{N I C}^{a n}$ affects the input impedance in port 1 . 


$$
\begin{aligned}
& \Delta \Gamma_{I N}=\frac{\left.\frac{\partial \Gamma_{I N}}{\partial \Gamma_{N I C}}\right|_{\Gamma_{N I C}=\Gamma_{N I C}^{a n}} \cdot \Delta \Gamma_{N I C}=\operatorname{Sens} \cdot \Delta \Gamma_{N I C}}{\text { Sens }} \\
& \text { Sens }=\left.\left|\frac{s_{21} \cdot s_{12}}{\left(1-S_{12} \cdot \Gamma_{N I C}\right)^{2}}\right|\right|_{\Gamma_{N I C}=\Gamma_{N I C}^{a n}} \\
& =\left|\frac{\left(s_{11} \cdot s_{22}-S_{21} \cdot s_{12}\right)^{2}}{s_{21} \cdot s_{12}}\right|
\end{aligned}
$$

The two ports must be placed in such a way that the sensitivity is minimized. Otherwise, any small change in the impedance provided by the NIC will dramatically affect the antenna performance in terms of the impedance bandwidth at port 1 . The result of this analysis over our proposed antenna, as well as the antenna structure is depicted in Fig. 2a. The averaged interval of frequency to compute Sens is $20-500$ MHz.

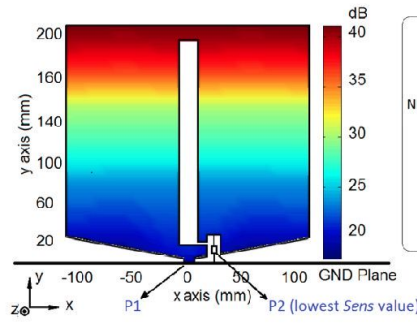

(a)

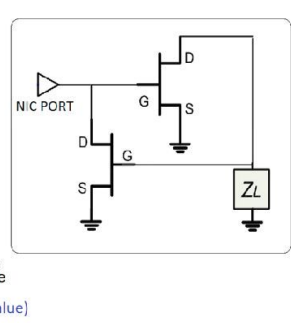

(b)
Fig. 2 a.) Sens parameter in $\mathrm{dB}$ over the blade monopole structure. b.) NIC topology selected for this design.

\section{ACTIVE VS PASSIVE MATCHING NETWORKS}

\section{A. Non foster matching network}

Once the best position for the port 2 is found, an inherently non-Foster circuit has to be chosen, as simple as possible for implementation feasibility, to emulate the effect of in the impedance matching, but avoiding a dramatic decrease in the radiation efficiency. A grounded NIC topology is selected and depicted in Fig. 2b. The active device is the BF998 FET. A fractional impedance bandwidth $(F B W)$ of $63 \%$ is obtained once the NIC is placed at port 2, as can be seen in Fig. 3. This result indicates a very high degree of miniaturization at the lower bound of the design interval since the factor $k a$ is equal to 0.18 (at $50 \mathrm{MHz}$ ), where $a$ is the radius of the smallest sphere enclosing the entire antenna system at the resonance frequency $\omega_{0}$, and $k$ is the free-space wavenumber (i.e. $k=$ $\left.\omega_{0} / c\right)$.

\section{B. Passive matching network}

With a conventional passive network (i.e. with positive inductors and capacitors) disposed as a cascade system, the best
$F B W$ obtained is just around $1.32 \%$, using a double series LC tank (see Fig. 3).

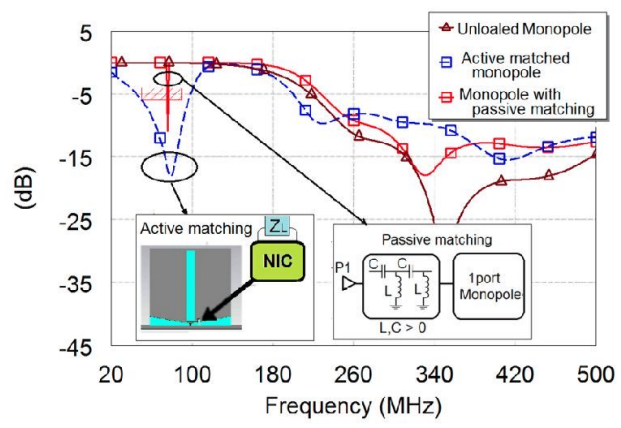

Fig. 3 Comparative response of the $\left|S_{11}\right|$ parameter.

\section{OTHER PERFORMANCE CONSIDERATIONS}

\section{A. Radiation pattern}

The simulated radiation pattern is depicted in Fig. 4. Two main lobes can be seen for the active MN case, but with no nulls. This kind of pattern would be acceptable for some aeronautical systems. A conventional monopole-like radiation pattern is observed for the passive MN case.

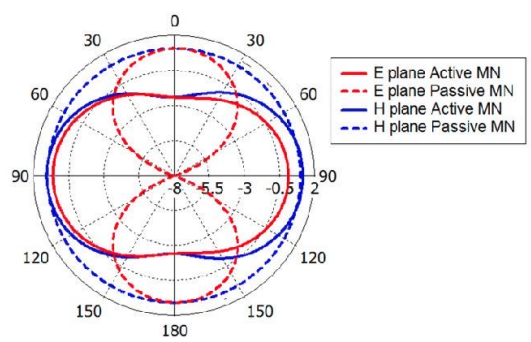

Fig. 4. Simulated radiation pattern of the active matched monopole

\section{B. Radiation efficiency}

In order not to affect the radiation efficiency, the NIC circuit realization must have a low resistive part (ideally zero). As the electrical size of the antenna decreases, this becomes to a very important parameter to take into account.

\section{REFERENCES}

[1] A.D. Yaghjian, S.R. Best, "Impedance, bandwidth, and Q of antennas," IEEE Trans. Antennas Propag., vol. 53, pp. 1298-1324, April 2005.

[2] E. Sussman-Fort, R.M. Rudish, "Non-Foster impedance matching of electrically-small antennas," IEEE Trans. Antennas Propag., vol. 57, pp. 2230-2241, August 2009.

[3] F. Albarracin-Vargas, E. Ugarte-Muñoz, V. Gonzalez-Posadas, D. Segovia-Vargas "Sensitivity analysis for active matched antennas with non-Foster elements," IEEE Trans. Antennas Propag., vol. 62, pp. 6040 6048 , December 2014 